\title{
Study on Wireless Sensor Network Localization Technology Based on DV-Hop Algorithm
}

\author{
Yong Yang \\ College of Electrical and Control Engineering, Xi'an \\ University of Science and Technology, Xi'an 710054, \\ P.R.China \\ guoxiuicai@163.com
}

\author{
Xinliang Zhang \\ Computer Science, Xi'an University of Science and \\ Technology, Xi'an 710054, P.R.China \\ 13772560698@163.com
}

\author{
Xiucai Guo \\ College of Electrical and Control Engineering \\ Xi'an University of Science and Technology \\ Xi'an 710054, P.R.China \\ guoxiucai@163.com
}

\begin{abstract}
DV-Hop algorithm is one of the important range-free localization algorithms. According to the localization principle of the DV-Hop algorithm and the defect of wireless sensor networks, this paper proposes three improvements including the estimation of average single hop distance, generalizing average single-hop distance and refining estimated results. This also simulated the performance of these improvements in different circumstances. The results show that the proposed improvements can greatly enhance the localization accuracy of the unknown nodes.
\end{abstract}

Keywords- Range-free; Sensor network; DV-Hop, Node localization

\section{INTRODUCTION}

Sensor network is a network made up by group of sensors which constitute a wired or wireless network by the Ad Hoc way. Its aim is to obtain the information generated by the sensors covering the monitored area then send them to the observers ${ }^{[1]}$. The self-positioning technology as the key technologies on the node have great significances on the application of wireless sensor networks and location-based network protocol.

Today the GPS location technology is mature. But GPS can not provide good positioning accuracy for some reason, and Most of the energy of sensor network nodes restricts the use of GPS. In addition, when considering the thousands of nodes, GPS devices will become extremely expensive to install. So, in recent years, people developed a number of location technologies which without need the GPS helps. According to the need or not to use the process of measuring the actual distance between nodes, these technologies can be divided into the localization algorithm based on distance and range-free localization algorithm. Distance-based algorithms need to measure the positioning or the absolute distance between adjacent nodes, and use of measurement information to calculate the location of unknown node.
However, the range-free algorithm use of network connectivity and estimated distance between nodes to calculate the location of unknown node. Compared to the algorithm based on distance, the range-free algorithm have 1 low cost, power consumption, simple hardware device, and most applications can be provided an acceptable accuracy of the positioning. Currently, the main location algorithms are Centroid algorithm, Convex programming algorithm, DVHop algorithm, Amorphous algorithm, MDS-MAP algorithm, APIT algorithm.

In the above algorithm, the DV-Hop algorithm has the advantages of simple and high positioning accuracy. DVHop localization algorithm is divided into 3 phases. Firstly, Using a distance vector routing exchange protocol, make all unknown nodes in the network to obtain the minimum distance and hop count from all reference nodes. Secondly, after each reference node has obtained the position and the hop count of the other reference nodes, calculate a singlehop distance based on themselves, and broadcast it to the network as a correction. Unknown node only record the first receiving number of each average single-hop distance and sent to neighbor nodes. Then, every unknown node use the product of the each single hop distance and hop count to calculate their distance from every reference nodes. Finally, after the unknown node get at least 3 reference nodes distance, it calculate the location using Maximum likelihood estimation, Trilateration or Centroid algorithm.

DV-Hop algorithm under the rules of distribution network has a good positioning effect, but on Irregular network topology wireless sensor networks, it is relatively large positioning error ${ }^{[2]}$. So, how to reduce the node localization error on irregular topology network has become the focus to research by people who study on wireless sensor networks. And there are some improvements on it. For example, there is improvement in two areas from the average single-hop distance and node connectivity constraints $^{[3]}$. And there is some one adding number 
threshold and weight threshold of hop count in DV-Hop second stage ${ }^{[4]}$. And some one use a loop of refinement to improve the performance of the algorithm ${ }^{[5]}$ etc..

This paper based on the localization process of DV-Hop algorithm proposed ways to improve in three areas. (1) Calculate the average single distance using minimum mean square method. (2) Generalize the single hop distance correction. (3) Refine the estimate result using the least squares based on Taylor expansion. Computer simulations show that, the algorithm improved in this paper is not only simple and high precision but also speeding up the convergence rate of refinement and saving energy resources of nodes.

\section{INTRODUCTION TO DV-HOP ALGORITHMS}

Figure 1 is a wireless sensor network formed by a few nodes. Where, $\mathrm{L}_{1}, \mathrm{~L}_{2}, \mathrm{~L}_{3}$ for the three reference nodes, the rest is unknown nodes. Here the unknown node $A$, for example, to illustrate the process of DV-Hop localization algorithm.

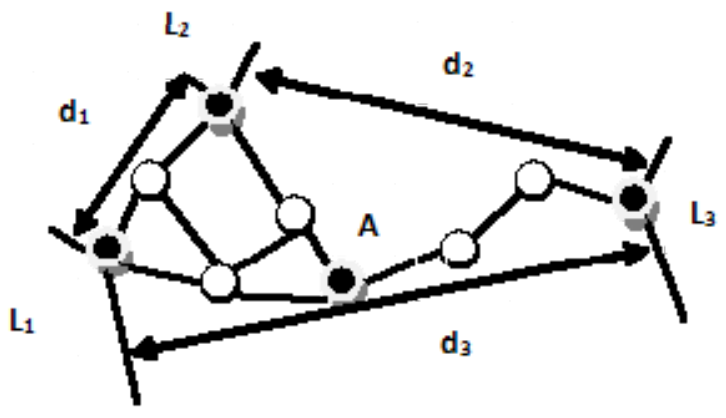

Fig.1 wireless sensor network formed by a few nodes

The first stage, Reference node broadcasts to the neighbors the self location information packet. The packet include hop number field and set the initial hop number 0 . Receiving nodes record the minimum hop number to each reference node and ignore the same packet which has the large hop number from the same reference node, then plus the packet's hop number 1 , and forwarded to neighbors. Of A, its hop number to the reference node to the reference node are 3,2,3.

The second stage, each reference node according to the records of the location information and the hop number information of the other reference nodes, each reference node according to the records of the location information and the hop number information of the other reference nodes, using the following formula estimates the average distance per hop.

$$
C K D_{i}=\sum_{i \neq j} d_{i, j} / \sum_{i \neq j} h_{i, j}
$$

Where, $\quad d_{i, j}=\sqrt{\left(x_{i}-x_{j}\right)^{2}+\left(y_{i}-y_{j}\right)^{2}}, \quad\left(x_{i}, y_{i}\right) \quad$ is the coordinates of $i, h_{i, j}$ is the hop number of reference node $i$ and reference node $\mathrm{j}(i \neq j), \mathrm{CKD}_{\mathrm{i}}$ is the single hop distance based the node $i$. In figure 1 , its hop number to each reference node are the single hop distance of each reference

are: $C K D_{1}=\left(d_{1}+d_{3}\right) /(2+6) \quad, \quad C K D_{2}=\left(d_{1}+d_{2}\right) /(2+5)$, $C K D_{3}=\left(d_{2}+d_{3}\right) /(5+6)$.

After that, each reference node broadcast the single hop distance to the network. Unknown nodes record the first receiving information packet and forwarded to neighbors. So, every unknown node can get the single hop distance from the every reference node from the nearest reference node. They can calculate their total distance to the reference node using the following formula.

$$
d_{i, k}=C K D_{i} \times h_{i, k}
$$

So, of $A$, the total distance to $\mathrm{L}_{1}, \mathrm{~L}_{2}, \mathrm{~L}_{3}$ are $d_{1, A}=C K D_{2} \times h_{1, A}=C K D_{2} \times 3, d_{2, A}=C K D_{2} \times h_{2, A}=C K D_{2} \times 2$ $d_{3, A}=C K D_{2} \times h_{3, A}=C K D_{2} \times 3$.

The third stage, unknown node based on total distance use trilateration or maximum likelihood estimation method to calculate their own coordinates.

\section{IMPROVEMENT ON DV-HOP ALGORITHM}

This section will be improvements in three aspects, include distance estimation and localization in the second stage and refinement of estimate values in the third stage.

A. Minimum mean square error method for solving the average single-hop distance (DV-hop(A))

Traditional methods are based on unbiased criteria to calculate the average single-hop distance $\mathrm{CKD}_{\mathrm{i}}$, through the following formula (3) value of zero to obtain.

$$
f_{1}=\frac{1}{N-1} \sum_{i \neq j}\left(d_{i, j}-C K D_{i} \times h_{i, j}\right)
$$

Where $\mathrm{N}$ is the number of reference nodes. In general, the value Using (3) make the estimate error by Gaussian. According to estimation theory, as the cost function to estimate the sub error, mean square error used here is more reasonable than the variance or deviation only ${ }^{[6]}$. So, this paper uses the minimum mean square error method to estimate the average single-hop distance (4).

$$
f_{2}=\frac{1}{N-1} \sum_{i \neq j}\left(d_{i, j}-C K D_{i} \times h_{i, j}\right)^{2}
$$

Set $\frac{\partial f_{2}}{\partial C K D_{i}}=0$ for the following formula (5).

$$
C K D_{i}=\sum_{i \neq j}\left(h_{i, j} \times d_{i, j}\right) / h_{i, j}{ }^{2}
$$

This paper studied on the performance of this improvement method by computer simulation experiments. In experiment, here set the true location of unknown node $C_{i}$, set the estimated coordinate ${ }^{X_{i}}, \mathrm{r}$ is communication radius, set $\left|X_{i}-C_{i}\right|$ of a network simulation positioning error 
of unknown Node i, define $r_{1}=\sum_{i \neq j}^{M}\left|X_{i}-C_{i}\right| / M$ (M is the number of unknown node), define error $=r_{1} / r$ of the normalized average location error of Communication radius.

Experimental environment 1: here are 150(which contain reference nodes) random laying nodes in $100 \mathrm{~m} * 100 \mathrm{~m}$ area, $\mathrm{r}=16 \mathrm{~m}$, and studying the performance of localization of the reference nodes number from 5 to 25, plus 5 per time. In the Experiment results figures, the abscissa is the number of reference nodes, the vertical axis is normalized average error.

Experimental environment 2: here are 20 reference nodes in $100 \mathrm{~m}^{*} 100 \mathrm{~m}$ area, $\mathrm{r}=16 \mathrm{~m}$, studying the performance of localization of the total nodes number from 140 to 180, plus 10 per time. In the Experimental results figures, the abscissa is the total number of the network nodes (including the reference node), the vertical axis is normalized average error.

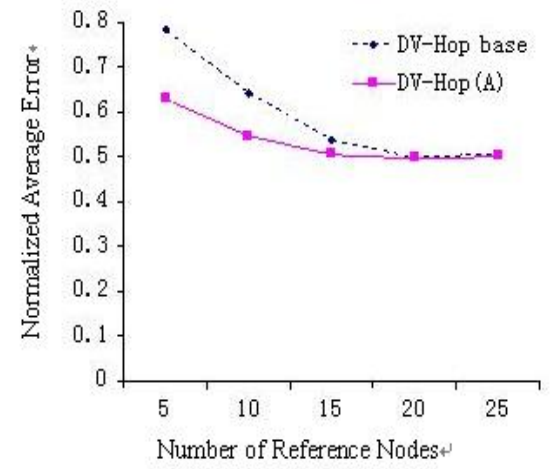

Fig.2 Experimental environment 1

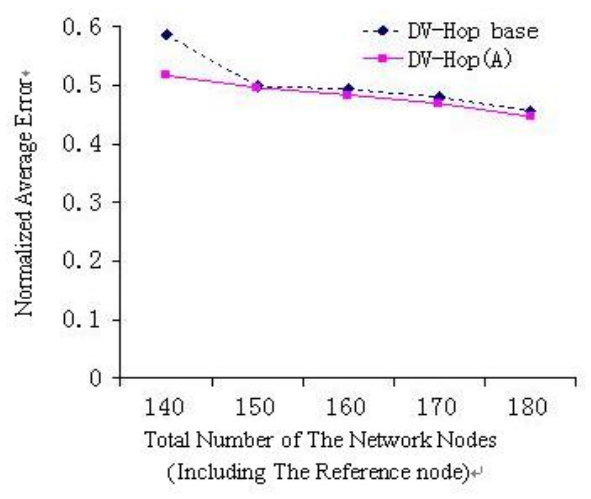

Fig.3 Experimental environment 2

The experimental data can show that: the method using mean square error can improve the precision of $5 \%$ whether in experimental environment 1 or experimental environment 2. It also can be drawn that whit the total nodes number increasing the location error will become increasingly smaller, and when the total number was a certain threshold, improved performance of the algorithm is relatively limited.

\section{B. Generalizing the average single-hop distance (DV-} $\operatorname{Hop}(B))$

In the Random wireless sensor networks, positioning accuracy is by the constraints of network topology. When there are empty areas in network, the signal transmission path must bypass the holes. This will cause the estimates based on single-hop distance and actual distance varies greatly. This is a common problem based on multi-hop localization algorithms.

Therefore, this paper will average all reference nodes per distance to a new value $C K D^{2}{ }_{i}$. Using this common and widespread value there will theoretically more help to improve the estimation accuracy.

$$
C K D^{2}{ }_{i}=\sum_{i \neq j}^{N} C K D^{1}{ }_{i} / N
$$

The following experiments will be in the experimental environment 1 and in the experimental environment 2 to study the improved performance.

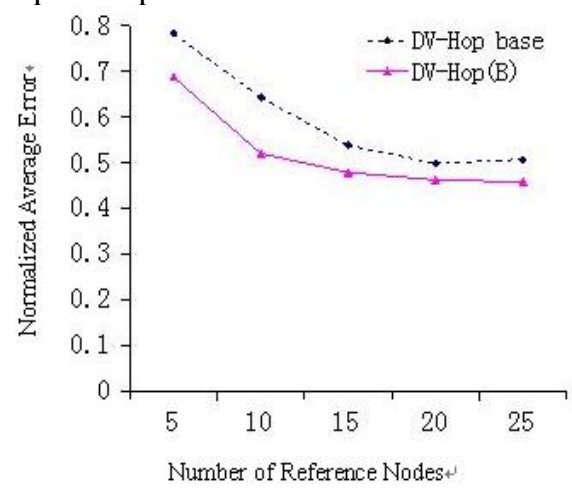

Fig.4 Experimental environment 1

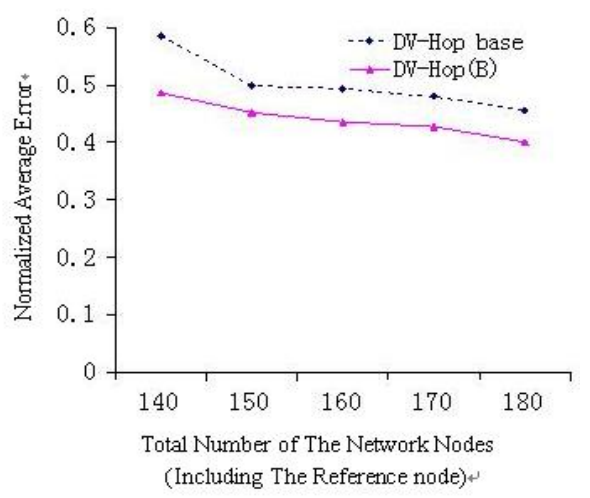

Fig.5 Experimental environment 2

Through this simulation we can see that, with the DVHop (B) Algorithm, the positioning accuracy have been increased obviously than DV-Hop base on unknown node.

C. Refine the estimated value using the least squares based on Taylor expansion $(D V-H o p(C))$

In the third stage of DV-Hop, the values which obtained within maximum likelihood estimation have large error, 
That can not meet the requirements of most applications. So, this stage here will improve the positioning accuracy by using the least squares based on Taylor expansion.

This refinement of the design steps are as follows.

(1) Establish the equations based on Taylor expansion and get ht, kt usage least squares. Of that, $\left(x_{i}, y_{i}\right)$ $(\mathrm{i}=1,2,3 \ldots \mathrm{N})$ is the coordinates of each reference node. About $\left(x_{0}, y_{0}\right)$, its initial value is its maximum likelihood estimate.

$$
\left\{\begin{array}{l}
\frac{\left(x_{0}-x_{1}\right)}{\sqrt{\left(x_{0}-x_{1}\right)^{2}+\left(y_{0}-y_{1}\right)^{2}}} h t+\frac{\left(y_{0}-y_{1}\right)}{\sqrt{\left(x_{0}-x_{1}\right)^{2}+\left(y_{0}-y_{1}\right)^{2}}} k t=d_{1}-\sqrt{\left(x_{0}-x_{1}\right)^{2}+\left(y_{0}-y_{1}\right)^{2}} \\
\frac{\left(x_{0}-x_{2}\right)}{\sqrt{\left(x_{0}-x_{2}\right)^{2}+\left(y_{0}-y_{2}\right)^{2}}} h t+\frac{\left(y_{0}-y_{2}\right)}{\sqrt{\left(x_{0}-x_{2}\right)^{2}+\left(y_{0}-y_{2}\right)^{2}}} k t=d_{2}-\sqrt{\left(x_{0}-x_{2}\right)^{2}+\left(y_{0}-y_{2}\right)^{2}} \\
\frac{\left(x_{0}-x_{n}\right)}{\sqrt{\left(x_{0}-x_{n}\right)^{2}+\left(y_{0}-y_{n}\right)^{2}}} h t+\frac{\left(y_{0}-y_{n}\right)}{\sqrt{\left(x_{0}-x_{n}\right)^{2}+\left(y_{0}-y_{n}\right)^{2}}} k t=d_{n}-\sqrt{\left(x_{0}-x_{n}\right)^{2}+\left(y_{0}-y_{n}\right)^{2}}
\end{array}\right.
$$

(2)Usage of the step value ht, kt to adjust the loop

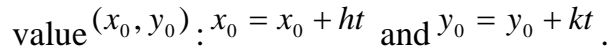

(3) Loop to step (1) (2) three times.

The value $\left(x_{0}, y_{0}\right)$ obtained finally is the final estimate of the improvement algorithm.

The following experiments will be in the experimental environment 1 and in the experimental environment 2 to study the improved performance.

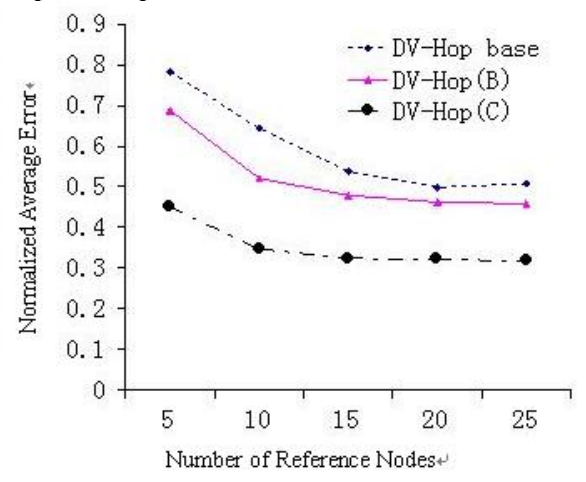

Fig.6 Experimental environment 1

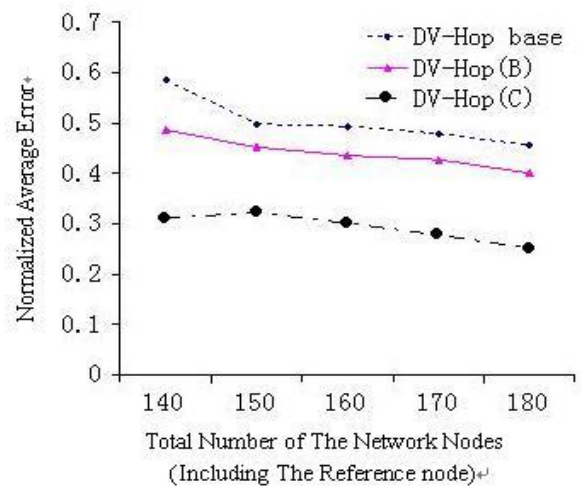

Fig.7 Experimental environment 2

Through the above experiment can be seen that, DVHop(C) algorithm can allows nodes to improve the average accuracy greater than $40 \%$ from DV-Hop base

\section{EXPERIMENTAL FINDINGS}

In the stage of $\mathrm{A}$, as calculating whit the method of minimum mean square error method for solving the average single-hop, it can improve the positioning accuracy of about $5 \%$. And that is no increase in the phase of the improvement of computational of nodes, no additional burden to the network.

In the B stage, we use a generalization of the network correction to solve the incompatibility of sensor network of random topological characteristics and DV-Hop algorithm. This method improves the positioning accuracy of about $10 \%$.

In the stage of $\mathrm{C}$, the process of refinement made the average accuracy increase of about $40 \%$. In this stage, on one hand, the initial value $\left(x_{0}, y_{0}\right)$ was the maximum likelihood estimation value; the other hand, the step size in loop calculations was extended. Experiments shows that, DV-Hop(C) can make $\sqrt{h t^{2}+k t^{2}} \leq 0.1$ and not only reduce the times of loop calculate but also can speed up the convergence rate of refinement.

\section{CONCLUSION}

To improve the DV-Hop algorithm in wireless sensor networks to locate the performance, this paper proposed 3point improvement: (1) Minimum mean square error method for solving the average single-hop distance; (2) Generalizing the average single-hop distance; (3) Refine the estimated value using the least squares based on Taylor expansion. The simulation experiments show that the proposed refinement method in this paper not only improves the accuracy but also saves the energy of the node. So that the cost of the networks in less, good localization results obtained.

\section{REFERENCES}

[1] Tilak S,Abu-Ghazaleh NB,Heinzelman W.A taxonomy of wireless micro-sensor network models[J].Mobile Computing and communications Review,2002,1(2):1-8

[2] Niculescu D and Nath B. Ad-hoc positioning system(APS). Proc. of the IEEE GIOBECOM, San Autonio, 2001: 2926-2931

[3] Ji Weiwei, Liu Zhong, Study on the Application of DV-Hop Localization Algorithms to Random Sensor Networks [J], Journal of Electronics \& Information Technology, 2008, 30(4): pp. 970-974

[4] Yu jin, Study on Multi-hop Positioning Mechanism and Energy Saving Coverage Technology, Master of Engineering Thesis [R], Wuhan University of Technology, 2008.5

[5] Yu Ning, Wan Jiangwen, etc., Localization Refinement Algorithms for Wireless Sensor Networks [J], Chinese High Technology Letters, 2008, 18(10), 1017-1022

[6] Zhang Xianda, Modern Signal Processing [M], Tsinghua University Press, 2002, 40-42 